\title{
Paediatric renal transplantation
}

\author{
Chandra Abeysekera ${ }^{1}$ \\ Sri Lanka Journal of Child Health, 2007; 36: 16-21
}

(Key words: renal transplantation, children)

From its early days as an experimental mode of treatment, renal transplantation has today become the treatment of choice for children with end stage renal disease $(E S R D)^{1}$. While great advances have been made in the field of renal replacement therapy for children with end stage renal failure, the provision of long term dialysis and associated therapies place major restrictions on the child and family. Poor weight gain and linear growth may necessitate supplementary feeding and/or recombinant growth hormone and further dietary restrictions are imposed by potassium and phosphate restricted diets that are necessary. Children also require oral and subcutaneous drug therapy for the prevention and treatment of anaemia, renal osteodystrophy and hypertension. Retardation of psychomotor development affects normal childhood activities; interferes with schooling, haemodialysis and peritoneal dialysis.

The restoration of normal or near normal renal function by renal transplantation allows moving away from these difficult and time consuming therapies and may facilitate better growth and neurodevelopment. Furthermore, adult data clearly shows transplantation to be more cost effective and to be associated with a reduction in long term mortality and improvement in quality of life compared to long term dialysis ${ }^{1}$.

In the early days of paediatric renal transplantation, less data were available to guide the specific care of infants and children who received a renal allograft and many approaches were derived by inference from experience in adult transplantation. Although some success was achieved with this approach, it overlooked the differences in immune responsiveness, drug metabolism and clearance, perfusion of transplanted kidneys and special needs relating to growth and development in children as compared to adults. Compared with large adult

${ }^{1}$ Senior Lecturer, Head, Department of Paediatrics, University of Peradeniya transplant programmes, individual paediatric centres were involved in a small number of paediatric transplants and clinical studies in paediatrics were small and uncontrolled. Collaborative studies and data registry systems overcame this problem and allowed better understanding and advanced paediatric renal transplantation. Several database registries currently collect data on paediatric renal transplantation. It includes United Network of Organ Sharing (UNOS), United States Renal Data System (USRDS) $^{2}$, North American Paediatric Renal Transplant Cooperative Society (NAPRACTS) ${ }^{3}$ and UK Renal Registry.

\section{Indications for renal transplantation}

Renal replacement therapy is indicated when significant complications from chronic renal insufficiency are present. These complications include symptoms of uraemia not responsive to standard medical therapies, failure to thrive due to limitations in calorie intake, delayed psychomotor development, hypervolaemia, hyperkalaemia and metabolic bone disease due to renal osteodystrophy ${ }^{4}$. The urgency and timing of renal transplantation in children must be considered in the context of a number of issues unique to children with Chronic Kidney Disease (CKD) such as delayed cognitive and educational performance, growth retardation, delayed puberty, aetiology of ESRD and timing of immunizations.

There are only a few reasons to withhold transplantation from children with $\mathrm{ESRD}^{4}$.

\section{Absolute contraindications to transplantation are:}

- Active or untreated malignancy.

- Chronic HIV infection.

- Chronic active infection with hepatitis B.

- Severe multi-organ failure that precludes a combined transplant with a kidney.

- Positive direct cross match or positive direct cross match within the previous 12 months.

- Debilitating, irreversible brain injury. 


\section{Relative contraindications to transplantation are:}

- $\mathrm{ABO}$ incompatibility with the donor.

- Ongoing active disease such as haemolytic uraemic syndrome or rapidly progressive glomerulonephritis.

- Active auto-immune disease such as systemic lupus erythematosus.

- Psychomotor retardation or psychiatric illness of such severity that custodial care is required.

- Chronic infection with hepatitis $\mathrm{C}$ virus.

- Serious long standing non-compliance with medical management.

- Lack of adequate home supervision or family support for a transplant patient.

\section{Pre-transplant evaluation of the recipient}

Pre-transplant evaluation is undertaken to ensure the patient's suitability as a transplant recipient. A thorough history, physical examination, determination of the blood group, HLA typing, biochemical and haematological evaluation, screening for exposure to infections, serological studies and radiological evaluation, including Doppler studies for adequate vascular patency, are necessary.

The cause for ESRD should be determined if possible ${ }^{1,2}$. In children, primary renal diseases responsible for ESRD are mostly congenital and hereditary disorders. Abnormalities of the urinary tract and bladder dysfunction are common contributing factors to ESRD in children. In many instances, bladder dysfunction that contributes to ESRD also contributes to allograft dysfunction and it is important to work closely with a urologist during the transplant evaluation to clearly define any possible interventions to maximize bladder function before transplantation. Many will have recurrent urinary tract infections requiring pretransplant native nephrectomy to prevent continued infections after transplantation. In addition, these children frequently display various inherited and sporadic syndromes with multiorgan involvement requiring the expertise of a variety of sub specialists.

The potential for recurrence of some pathologic lesions, such as focal segmental glomerulosclerosis (FSGS), atypical haemolytic uraemic syndrome (HUS) and membranoproliferative glomerulonephritis should be considered and strategies developed to monitor recurrences and treatment. In some situations, bilateral native nephrectomy and short term dialysis should be considered before transplantation to minimize problems after transplantation. Clinical conditions that require consideration of pre-transplantation native nephrectomy include polyuria, severe electrolyte wasting, massive proteinuria, recurrent pyelonephritis and hypertension ${ }^{5}$.

The child should receive age appropriate immunizations including hepatitis $\mathrm{B}$ and varicella ${ }^{6}$. Nutritional status should be optimized. Some children need cardiac, pulmonary, dental and ophthalmic evaluation. In addition, the child should be assessed by a nephrologist, surgeon, urologist, anaesthetist, coordinator, dietician and a social worker.

In developing countries, socioeconomic factors should be carefully considered before committing infants and children for transplantation in the light of financial constraints of providing life long immunosuppressive drugs. The family support system should be explored and strengthened. The child and family's ability to comply with immunosuppressive and medical therapy cannot be underestimated as non compliance is the single most important cause for graft failure, particularly in adolescents.

\section{Pre-emptive transplantation}

Because renal transplantation has become a more routine procedure, many patients receive a renal allograft before reaching ESRD and the need for dialysis therapies. The benefits and detriments to these approaches have been debated for many years. Although pre-emptive transplantation presents an opportunity to limit the period of uraemia as well as to avoid procedures which may permanently compromise blood vessels or result in injury to the peritoneal membrane, some researchers have suggested that it does not allow the maximum use of native kidney function, nor take advantage of the immunosuppression of uraemia or provide experience of dialysis as a stimulus to reinforce compliance with a medical regimen. However, pre-emptive transplantation avoids the mortality and morbidity associated with dialysis and recipients of pre-emptive transplantation are shown to have significantly improved patient and allograft survival ${ }^{3,7,8}$. Therefore, every effort must be made to improve recognition of impending ESRD to allow time for patient evaluation and preparation and family discussions plus location of potential donors to maximize the use of preemptive transplantation. 


\section{Donor evaluation}

The transplant donors can be either living related, unrelated or deceased donors. Many family members, as well as friends, often volunteer to be a donor for a child. These offers are usually emotion based. It is important that adequate evaluation of the donor be done. In order to avoid any conflict of donor and recipient selection, in most paediatric programmes the donor is evaluated by an adult -based group who would not be taking care of the child.

Psychologically the donor must be emotionally prepared to undergo an evaluation. ABO compatibility needs to be maintained to achieve a successful donation. Rhesus matching is generally not considered. HLA matching is often used to identify optimal donors for paediatric patients. Data to date suggest that an 0 -antigen mismatch (perfect match) kidney has an improved outcome over a one haplotype mismatched kidney ${ }^{9}$. $\mathrm{T}$ and $\mathrm{B}$ cell antibodies should be screened in the donor and recipient independent of the donor source, cadaveric or living ${ }^{9}$. The standard of care is to not transplant in face of a positive $\mathrm{T}$ cell cross match with less clear data on the B cell cross match.

In order to donate a kidney the donor must obviously be in good health. It is imperative to ensure that the donor operation does not impair the donor's health. Obvious issues, such as having two kidneys, as well as having no underlying renal vascular disease, are prerequisites.

The donor should undergo a complete physical examination looking for potential risk factors related to organ donation. This would include a 24-hour urine creatinine clearance, 24 hour urine protein excretion, 24 hour ambulatory blood pressure monitoring and signs of cardiac, pulmonary or hepatic disease. It is important to identify any underlying premalignant or malignant conditions. The donor also needs to understand the economic impact of donation.

All donors, living and cadaveric, need to be screened for infectious diseases including HIV, hepatitis A, B, $\mathrm{C}$ and tuberculosis as well as other potential communicable diseases. Donor evaluation of cytomegalovirus (CMV) and Epstein Barr virus (EBV) status should become standard.

Radiological evaluation of the donor's kidney may include the use of ultrasound, DTPA scan, angiogram, IVU, spiral CT or MRA depending on the local standard of practice. The absolute requirements for a transplant are a healthy donor with functioning two kidneys, compatible blood group and a negative cytotoxic cross match. While HLA matching is not a requirement, HLA identical grafts have a longer halflife compared to those that are less well matched.

\section{Immunosuppression}

During the early days of transplantation, the choices of immunosuppressive drugs were few and most patients were treated similarly. The last decade has seen an explosion of new immunosuppressive medications which have presented a new challenge to the transplant physician to understand the clinical effects, side effects and interactions of a larger array of medications.

Immunosuppressive medications currently used include

- Corticosteroids

- Antiproliferative drugs - azathioprine, mycophenolate mofetil, sirolimus

- Calcineurin inhibitors - cyclosporine, tacrolimus

- Biologic

○ Polyclonal antibodies - antithymocyte globulin

- Monoclonal antibodies - OKT 3, IL 2 receptor blockade (basiliximab, daclizumab)

The choice of immunosuppressive therapy for the recipient is based on the local standard of care. This may be either cyclosporine based or tacrolimus based protocols. Children treated with tacrolimus based triple therapy were shown to have a reduced incidence of acute rejection and a significantly improved GFR at one year post transplantation compared with those treated with cyclosporine based therapy.

\section{Complications of transplantation}

The complications that can occur following transplantation include complications related to surgery, allograft rejection, delayed allograft function, infections, hypertension, metabolic and haematological complications and the recurrence of primary disease in the allograft. There are other miscellaneous complications including side effects of medications, excessive weight gain, growth retardation, insulin dependant diabetes mellitus, osteopenia, delayed allograft rejection, chronic allograft dysfunction and malignancies. 


\section{Acute rejections}

Acute rejection is the most common cause for renal allograft loss. However, with improved surgical techniques, understanding of the immunologic process involved in transplantation and growing number of immunosuppressive agents available, there has been a marked reduction in the frequency of rejections in children. In addition, current approaches to treatment of rejection have been associated with improvement of rejection reversal. However, acute rejection continues to be a significant problem and the second leading cause of graft loss ${ }^{10,11}$.

\section{Allograft survival}

Registries held in the United Kingdom and in North America provide good quality data on long term survival. A one year allograft survival rate in the period $1996-2000$ is $96 \%$ for live donor and $94 \%$ for cadaveric donor sources respectively. There has been a similar improvement in long- term allograft function. Allograft half life ( $\mathrm{T} 1 / 2$ ) has improved from 15.4 in a live donor (LD) and 9.5 in a cadaveric donor.(CD) in 1989 to 25.4 (LD) and 16.4 (CD) in 1996. No single factor can be identified as the source for this improvement in graft survival, but improved surgical techniques or immunosuppressive therapy would have been contributory.

\section{Factors influencing graft outcome}

Data registries have identified several factors that affect the outcome of grafts in children. These include younger donor age, younger recipient age, poor HLA matching, long cold ischaemia time and ethnicity. Cold ischaemia time refers to the time that the harvested organ is stored in ice after having been perfused with organ preservation fluid. Graft outcome deteriorates with increasing storage time, the risk of graft failure at three months posttransplantation increasing by $4 \%$ with each additional hour of cold ischaemia time. It is well established that poor HLA matching results in both a reduction in long term graft survival and an increased risk of immunological sensitization (anti-HLA antibody production) and reduces the number of potential donor organs suitable for future transplantation. The UKT study showed that o, o, o mismatched and favourably matched kidneys were associated with the best transplant outcome.

\section{Graft failure and patient mortality}

While the short and long term outcome of transplantations continues to improve, graft failure remains a substantial problem. The rate of graft loss is the highest in the first year following transplantation and a majority of these kidneys are lost in the first month or two from vascular events and acute rejections. Thereafter, the rate of graft loss slows. Of the 582 grafts that have failed in the UKT analysis and the 1592 grafts that have been lost in the NAPRACTS series (1987 onwards) rejection has been the commonest cause for graft loss, accounting for $50.4-55.5 \%$ of all losses. There were $19.3 \%$ acute rejections and $31.1 \%$ chronic rejections in NAPRACTS series. Vascular thrombosis was responsible for $10-12.8 \%$ of all losses, the majority occurring in the immediate post- transplant period. 7$9 \%$ losses occurred due to death with a functioning transplant ${ }^{11}$.

The overall mortality rate in the 12th Annual NAPRACTS report was $6.5 \%$ in the first five years post transplantation. Infections and cardiopulmonary events were the commonest causes of death in both this and the UKT series. It is becoming increasingly apparent that cardiovascular disease, which is the cause of death in a large proportion of adult kidney recipients, is also a very significant problem in the paediatric and young adult population ${ }^{12}$.

As is common in many childhood chronic conditions, non-compliance with medication is a major problem in transplanted patients, the problem being greatest amongst the adolescent population ${ }^{13}$. Non compliance with immunosuppressive therapy is the commonest cause for late graft loss, with $15-16 \%$ of children losing their grafts due to this ${ }^{14,15}$. The problem appears to be greatest with those medications which are complex to administer or are associated with adverse side effects. Cosmetic side effects, particularly the cushingoid facies and acne induced by corticosteroids, and the hypertrichosis and gingival hyperplasia induced by cyclosporine are a particular source of distress to some children and are known to be linked to an increased rate of noncompliance. Despite the major clinical and economic implications of non-compliance, this remains a vastly under-researched and under-resourced area in medicine.

\section{Summary}

Renal transplantation is a richly rewarding endeavour since it transforms chronically ill children into near normal children in a matter of weeks to months with an improvement in their neurodevelopment and quality of life. There have been remarkable advances in renal transplantation over the past two decades with reduced morbidity and mortality, reduced 
rejection rates and improved long term patient and allograft survival. The future of transplantation continues to be exciting, with opportunities for reduced immunosuppressive medications and their side effects and the elusive goal of transplantation tolerance seems to be within reach. However, in developing countries, socioeconomic factors should be carefully considered before committing infants and children for transplantation in the light of financial constraints of providing life long follow up and immunosuppressive drugs.

\section{References}

1. Wolfe RA, Ashby VB, Milford DV, et al. Comparison of mortality in all patients on dialysis, patients on dialysis awaiting transplantation and recipients of a first cadaveric transplant. N Engl J Med 1999; 341: 1725-30.

2. System USRD. USRDS 2002 annual data report: atlas of end-stage renal disease in the United States. Bethesda; National Institutes of Health, National Institute of Diabetes and Diabetes and Digestive and Kidney Diseases;2002.

3. Benfield MR, Mc Donald RA, Bartosh SM, Ho PL, Harmon WE, Changing trends in paediatric transplantation: 2001 annual report of the North American Paediatric Renal Transplant Cooperative Study. Paediatr 2003; 7: 321-35.

4. Davis ID, Bunchman TE, Grimm PC, et. al. Paediatric renal transplantation: indications and special considerations. A position paper from the Paediatric Committee of the American Society of Transplant Physicians. Paediatric Transplant 1998; 2(2):717-9.

5. Nahas WC, Mazzucchi E, Pinheiro MS, et al. Role of native nephrectomy in renal transplant recipients. Transplant Proc 2002; 34(2): 717-9.

6. Davis ID. Immunization in the transplant patient. Transplant Chronicles 1996; 3: 6-7.
7. Kasiske BL, Snyder JJ, Matas AJ, Ellison MD, Gill JS, Kausz AT. Pre-emptive kidney transplantation: the advantage and the advantaged. J Am Soc nephrol 200; 13(5): 135864.

8. Mehta A, Gulati S, Sharma RK, Kumar A. Preemptive transplant,a rational therapeutic approach. Indian Paediatr 2001; 38(10):1171-3.

9. Kasiske BL, Ravenscraft M, Ramos EL, gaston RS, Bia MJ, Danovitch GM, The evaluation of living renal transplant donors: clinical practice guidelines. Ad Hoc Clinical Practice Guidelines Subcommittee of the Patient Care and Education Committee of the American Society of Transplant Physicians. J Am Soc Nephrol 1996; 7: 2288-313.

10. Seilkaly M, Ho PL, Emmett L, Tejani A. The 12th annual report of the North American Paediatric Renal Transplant Cooperative Study: renal transplantation from 1987 through 1998. Paediatr Transplant 2001; 5(3):215-31.

11. Mc Donald R, Ho PL, Stablein DM, Tejani A, North American Paediatric Renal Transplant Cooperative S. Rejection Profile of recent paediatric transplant recipients compared with historical controls: a report of the North American Paediatric Renal Transplant Cooperative Study (NAPRACTS). Am J Transplant 2001; 1(1):55-60.

12. Aakhus S, Dahl K, Wideroe TE. Cardiovascular morbidity and risk factors in renal transplant recipients. Nephrol Dial Transplant 1999; 14: 648-54.

13. Meyers KE, Weiland $\mathrm{H}$, Thomson PD. Paediatric renal transplant non-compliance. Paediatr Nephrol 1995; 9: 189-92.

14. Korsch BM, Fine RN, Negrete VF. Noncompliance in children with renal transplants. Paediatrics 1978; 61:872-6.

15. Ettenger RB, Rosenthal JT, Marik JL, et.al. Improved cadaveric renal transplant outcome in children. Paediatr Nephrol 1993; 7:89-95. 